

\title{
Singular moduli that are algebraic units
}

\author{
Philipp Habegger
}

\begin{abstract}
We prove that only finitely many $j$-invariants of elliptic curves with complex multiplication are algebraic units. A rephrased and generalized version of this result resembles Siegel's theorem on integral points of algebraic curves.
\end{abstract}

\section{Introduction}

A singular modulus is the $j$-invariant of an elliptic curve with complex multiplication; we treat them as complex numbers in this note. They are precisely the values of Klein's modular function $j: \mathbb{H} \rightarrow \mathbb{C}$ at imaginary quadratic arguments; here $\mathbb{U}$ denotes the upper half-plane in $\mathbb{C}$. For example, $j(\sqrt{-1})=1728$. Singular moduli are algebraic integers and the set of all singular moduli is stable under ring automorphisms of $\mathbb{C}$. We refer to [Lang 1987] for such classical facts.

At the AIM workshop on unlikely intersections in algebraic groups and Shimura varieties in Pisa, 2011 David Masser, motivated by [Bilu et al. 2013], asked if there are only finitely many singular moduli that are algebraic units. Here we provide a positive answer to this question.

Theorem 1. At most finitely many singular moduli are algebraic units.

Our theorem relies on several tools: Liouville's inequality from diophantine approximation, Duke's equidistribution theorem [1988], its generalization due to Clozel-Ullmo [2004], and Colmez's lower bound [1998] for the Faltings height of an elliptic curve with complex multiplication supplemented by [Nakkajima and Taguchi 1991].

A numerical computation involving sage reveals that no singular modulus of degree at most 100 over the rationals is an algebraic unit. There may be no such units at all. Currently, there is no way to be sure as Duke's theorem is not known to be effective.

Below, we formulate and prove a general finiteness theorem reminiscent of Siegel's theorem on integral points on curves. We will see in particular that there are only finitely many singular moduli $j$ such that $j+1$ is a unit. Such $j$ do exist, since for instance $j((\sqrt{-3}+1) / 2)=0$ is a singular modulus.

MSC2010: primary 11G18; secondary 11G50, 11J86, 14G35, 14G40.

Keywords: elliptic curves, complex multiplication, heights. 
Suppose that $X$ is a geometrically irreducible, smooth, projective curve defined over a number field $F$. We write $F[X \backslash C]$ for the rational functions on $X$ that are regular outside of a finite subset $C$ of $X(F)$. Let $O_{F}$ be the ring of algebraic integers of $F$. A subset $M \subset X(F) \backslash C$ is called quasi-integral with respect to $C$ if for all $f \in F[X \backslash C]$, there exists $\lambda \in F \backslash\{0\}$ such that $\lambda f(M) \subset \mathcal{O}_{F}$. By clearing denominators one sees that quasi-integral sets remain so after adding finitely many $F$-rational points. Siegel's theorem, see [Serre 1997, Chapter 7], states that a quasiintegral set is finite if $C \neq \varnothing$ and the genus of $X$ is positive, or if the cardinality $\# C$ of $C$ is at least 3.

Our extension of Theorem 1 deals with the question of finiteness for quasi-integral sets of special points on modular curves. Special points generalize singular moduli, we provide a definition for them below. Only finitely many singular moduli are rational over a fixed number field. Thus we adapt the notion of quasi-integrality in the following way. Let $\bar{F}$ be an algebraic closure of $F$ and $\mathcal{O}_{\bar{F}}$ the ring of algebraic integers in $\bar{F}$. We again work with a finite set $C \subset X(\bar{F})$. A subset $M \subset X(\bar{F}) \backslash C$ is called quasi-algebraic-integral with respect to $C$ if for all $f \in \bar{F}[X \backslash C]$ there is a $\lambda \in \bar{F} \backslash\{0\}$ such that $\lambda f(M) \subset \mathrm{O}_{\bar{F}}$.

Let us recall some classical facts about modular curves. Let $\Gamma$ be a congruence subgroup of $\mathrm{SL}_{2}(\mathbb{Z})$, i.e., $\Gamma$ contains the kernel of the reduction homomorphism $\mathrm{SL}_{2}(\mathbb{Z}) \rightarrow \mathrm{SL}_{2}(\mathbb{Z} / N \mathbb{Z})$ for an $N \geq 1$. Then $\Gamma$ acts on $\mathbb{H}$, as does any subgroup of $\mathrm{SL}_{2}(\mathbb{R})$, by fractional linear transformations. The quotient $\mathbb{M} / \Gamma$ can be equipped with the structure of an algebraic curve $Y_{\Gamma}$ defined over a number field $F$. This algebraic curve has a natural compactification $X_{\Gamma}$, which is a geometrically irreducible, projective, smooth curve over $F$. The points of $X_{\Gamma} \backslash Y_{\Gamma}$ are called the cusps of $Y_{\Gamma}$. We remark that $Y(1)=Y_{\mathrm{SL}_{2}(\mathbb{Z})}$ is the affine line, that the compactification is $\mathbb{P}^{1}$, and that there is a single cusp $\infty$. The natural map $\phi: Y_{\Gamma} \rightarrow Y(1)$ is algebraic. A point of $Y_{\Gamma}(\bar{F})$ is called special if it maps to a singular modulus under $\phi$.

Theorem 2. Let $\Gamma \subset \mathrm{SL}_{2}(\mathbb{Z})$ be a congruence subgroup and $F \subset \mathbb{C}$ a number field over which $Y_{\Gamma}$ is defined. Let $C \subset X_{\Gamma}(\bar{F})$ be a finite set containing a point that is not a cusp of $Y_{\Gamma}$. Any set of special points in $Y_{\Gamma}(\bar{F})$ that is quasi-algebraic-integral with respect to $C$ is finite.

We require $C$ to contain a noncusp for good reason. Indeed, as singular moduli are algebraic integers, the set of all singular moduli is a quasi-algebraic-integral subset of $Y(1)(\overline{\mathbb{Q}})$ with respect to $C=\{\infty\}$. We recover Theorem 1 from Theorem 2 by taking $\Gamma=\mathrm{SL}_{2}(\mathbb{Z})$ and $C=\{0, \infty\}$. The proof of Theorem 2 relies on the same basic strategy as Theorem 1. However, instead of the Liouville inequality we require David and Hirata-Kohno's sharp lower bound for linear forms in elliptic logarithms [2009]. Earlier, Masser and others obtained lower bounds in this setting after A. Baker's initial work on linear forms in classical logarithms. 
Our theorems are similar to M. Baker, Ih, and Rumely's result [2008] on roots of unity that are $S$-integral relative to a divisor of $\mathbb{G}_{m}$. Indeed, both finiteness results are based on an equidistribution statement. However, the Weil height of a root of unity is zero, whereas the height of a singular modulus can be arbitrarily large. Indeed, the quality of Colmez's growth estimate for the Faltings height plays a crucial role in our argument. Moreover, finiteness need not hold in the multiplicative setting if the support of the divisor consists of roots of unity. This is in contrast to Theorem 1 where the support of the corresponding divisor is the singular modulus 0 . Finally, our work considers only the case where $S$ consists only of the Archimedean places whereas M. Baker, Ih, and Rumely also allow finite places.

Gross and Zagier [1985] gave a formula for the norm of the $j$-invariant of certain elliptic curves with complex multiplication. However, the author was unable to deduce the finiteness statement in Theorem 1 from their result or from Dorman's extension [1988].

Habegger would like to thank the organizers of the AIM workshop in Pisa, 2011, for providing a stimulation environment. He also thanks Su-ion Ih for helpful remarks concerning his paper with $\mathrm{M}$. Baker and Rumely.

\section{Unitary Singular Moduli}

In this section, $c_{1}, c_{2}, \ldots$ denote positive and absolute constants.

Let $K$ be a number field. A finite place $v$ of $K$ is a non-Archimedean absolute value that restricts to the $p$-adic absolute value on $\mathbb{Q}$ for some prime $p$. With this normalization we have $|p|_{v}=1 / p$. The completion of $K$ with respect to $v$ is a field extension of degree $d_{v}$ of the completion of $\mathbb{Q}$ with respect to the $p$-adic absolute value. Let $J$ be an algebraic number in a number field $K$. The absolute logarithmic Weil height of $J$, or just height for short, is

$$
h(J)=\frac{1}{[K: \mathbb{Q}]}\left(\sum_{\sigma} \log \max \{1,|\sigma(J)|\}+\sum_{\nu} d_{\nu} \log \max \left\{1,|J|_{\nu}\right\}\right)
$$

where $\sigma$ runs over all field embeddings $\sigma: K \rightarrow \mathbb{C}$ and $v$ runs over all finite places of $K$. It is well-known that $h(J)$ does not change when we replace $K$ by another number field containing $J$. For this and other facts on heights we refer to Sections 1.5 and 1.6 of [Bombieri and Gubler 2006].

We state a height lower bound for singular moduli that follows easily from a result of Colmez and of Nakkajima-Taguchi. See [Poonen 2001, Lemma 3] for a similar argument.

Lemma 3. Let $J$ be a singular modulus attached to an elliptic curve whose endomorphism ring is an order with discriminant $\Delta<0$. Then

$$
h(J) \geq c_{2} \log |\Delta|-c_{3} .
$$


Proof. We write $\Delta=\Delta_{0} f^{2}$ where $\Delta_{0}<0$ is a fundamental discriminant and $f$ is the conductor of the endomorphism ring of $E$, an elliptic curve attached to $j$. In Corollaire 7, Colmez [1998] proved (1) with $h(J)$ replaced by the stable Faltings height of $E$ when $\Delta$ is a fundamental discriminant, i.e., if $f=1$. For $f>1$ Nakkajima and Taguchi [1991] found that one must add

$$
\frac{1}{2} \log f-\frac{1}{2} \sum_{p \mid f} e_{f}(p) \log p
$$

to the stable Faltings height; here the sum runs over prime divisors $p$ of $f$ and

$$
e_{f}(p)=\frac{1-\chi(p)}{p-\chi(p)} \frac{1-p^{-n}}{1-p^{-1}}
$$

if $p^{n} \mid f$ but $p^{n+1} \nmid f$ and $\chi(p)$ is Kronecker's symbol $\left(\frac{\Delta_{0}}{p}\right)$. The arguments in the proof of [Habegger 2010, Lemma 4.2] give $\sum_{p \mid f} e_{f}(p) \log p \leq c_{1} \log \log \max \{3, f\}$. Therefore, the stable Faltings height of $E$ is logarithmically bounded from below in terms of $\left|\Delta_{0} f^{2}\right|=|\Delta|$.

By [Silverman 1986, Proposition 2.1], we can replace the stable Faltings height by $h(J)$ at the cost of adjusting the constants.

Our strategy to prove Theorem 1 is as follows. Let $J$ and $\Delta$ be as in Lemma 3 . Assume in addition that $J$ is an algebraic unit. We will find an upper bound for $h(J)$ that contradicts the previous lemma for sufficiently large $|\Delta|$. This will leave us with only finitely many $\Delta$ and hence finitely many $J$, as we will see.

The norm of $J$ is \pm 1 and the finite places do not contribute to the height of the algebraic integer $J$. Thus we can rewrite

$$
h(J)=\frac{1}{D} \sum_{|\sigma(J)|>1} \log |\sigma(J)|=-\frac{1}{D} \sum_{|\sigma(J)|<1} \log |\sigma(J)|
$$

where $D=[\mathbb{Q}(J): \mathbb{Q}]$ and where the sums run over field embeddings $\sigma: \mathbb{Q}(J) \rightarrow \mathbb{C}$.

For each $\sigma$ we have $\sigma(J)=j\left(\tau_{\sigma}\right)$ for some $\tau_{\sigma}$ in the classical fundamental domain

$$
\mathscr{F}=\{\tau \in \mathbb{H} ; \operatorname{Re}(\tau) \in(-1 / 2,1 / 2],|\tau| \geq 1 \text { and } \operatorname{Re}(\tau) \geq 0 \text { if }|\tau|=1\}
$$

of the action of $\mathrm{SL}_{2}(\mathbb{Z})$ on $\mathbb{H}$.

To bound the right-hand side of (2) from above we must control those conjugates $\sigma(J)$ that are small in modulus. Let $\epsilon \in(0,1]$ be a parameter that is to be determined; the $c_{i}$ will not depend on $\epsilon$. We define

$$
\Sigma_{\epsilon}=\{\tau \in \mathscr{F} ;|j(\tau)|<\epsilon\} .
$$


The field embeddings that contribute most to the height of $J$ are in

$$
\Gamma_{\epsilon}=\left\{\sigma: \mathbb{Q}(J) \rightarrow \mathbb{C} ; \tau_{\sigma} \in \Sigma_{\epsilon}\right\}
$$

We estimate their number using equidistribution in the next lemma.

Lemma 4. We have $\# \Gamma_{\epsilon} \leq c_{6} \epsilon^{2 / 3} D$ if $D$ is sufficiently large with respect to $\epsilon$.

Proof. Let $\mu$ denote the hyperbolic measure on $\mathscr{F}$ with total mass 1 , i.e.,

$$
\mu(\Sigma)=\frac{3}{\pi} \int_{x+y i \in \Sigma} \frac{d x d y}{y^{2}}
$$

for a measurable subset $\Sigma \subset \mathscr{F}$. Duke [1988] proved that the $\tau_{\sigma}$ are equidistributed with respect to $\mu$ as $\Delta \rightarrow-\infty$ runs over fundamental discriminants. For general discriminants, equidistribution follows from [Clozel and Ullmo 2004]. So, for $\Delta \rightarrow-\infty$, we get $\left|\# \Gamma_{\epsilon} / D-\mu\left(\Sigma_{\epsilon}\right)\right| \rightarrow 0$. To prove the lemma we will bound $\mu\left(\Sigma_{\epsilon}\right)$ in terms of $\epsilon$.

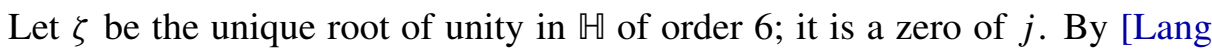
1987, Chapter 3, Theorem 2], Klein's modular function $j$ has a triple zero at $\zeta$ and at $\zeta^{2}$ and does not vanish anywhere else on $\overline{\mathscr{F}}$, the closure of $\mathscr{F}$ in $\mathbb{H}$. So $\tau \mapsto j(\tau)(\tau-\zeta)^{-3}\left(\tau-\zeta^{2}\right)^{-3}$ does not vanish on $\overline{\mathscr{F}}$. Now $j$ has a pole at infinity and so $|j(\tau)|>1$ if the imaginary part of $\tau$ is sufficiently large. Therefore

$$
|j(\tau)| \geq c_{4}|\tau-\zeta|^{3}\left|\tau-\zeta^{2}\right|^{3} \geq \frac{c_{4}}{8} \min \left\{|\tau-\zeta|,\left|\tau-\zeta^{2}\right|\right\}^{3}
$$

for all $\tau \in \overline{\mathscr{F}}$ with $|j(\tau)| \leq 1$ where $\max \left\{|\tau-\zeta|,\left|\tau-\zeta^{2}\right|\right\} \geq\left|\zeta-\zeta^{2}\right| / 2=\frac{1}{2}$ was used in the second inequality. Because the imaginary part of an element in $\mathscr{F}$ is at least $\sqrt{3} / 2$ we can use (3) to estimate $\mu\left(\Sigma_{\epsilon}\right) \leq c_{5} \epsilon^{2 / 3}$.

Using this lemma with (2) we can bound the height of $J$ from above as

$$
\begin{aligned}
h(J) & =-\frac{1}{D}\left(\sum_{|\sigma(J)|<\epsilon} \log |\sigma(J)|+\sum_{\epsilon \leq|\sigma(J)|<1} \log |\sigma(J)|\right) \\
& \leq c_{6} \epsilon^{2 / 3} \max _{|\sigma(J)|<\epsilon} \log \left(|\sigma(J)|^{-1}\right)+|\log \epsilon| .
\end{aligned}
$$

Soon we will use Liouville's inequality from diophantine approximation to bound $\left|j\left(\tau_{\sigma}\right)\right|$ from below if $\sigma \in \Gamma_{\epsilon}$. To do this, we require a bound for the height of $\tau_{\sigma}$.

Lemma 5. Each $\tau_{\sigma}$ is imaginary quadratic and we have $h\left(\tau_{\sigma}\right) \leq \log \sqrt{|\Delta|}$.

Proof. We abbreviate $\tau=\tau_{\sigma}$ and decompose $\Delta=\Delta_{0} f^{2}$ as in the proof of Lemma 3 . The endomorphism ring mentioned in Lemma 3 can be identified with $\mathbb{Z}+\omega f \mathbb{Z} \subset \mathbb{C}$ where $\omega=\left(\sqrt{\Delta_{0}}+\Delta_{0}\right) / 2$. This ring acts on the lattice $\mathbb{Z}+\tau \mathbb{Z}$. So there exist 
$a, b, c, d \in \mathbb{Z}$ with $\omega f=a+b \tau, \omega f \tau=c+d \tau$ and $b \neq 0$. We substitute the first equality into the second one and obtain

$$
b \tau^{2}+(a-d) \tau-c=0 .
$$

Hence, $\tau$ is imaginary quadratic. We note that $\omega f$ is a root of $T^{2}-(a+d) T+a d-b c$. The discriminant of this polynomial is $(a+d)^{2}-4(a d-b c)=(\omega-\bar{\omega})^{2} f^{2}=\Delta$. Hence $\tau=(-(a-d) \pm \sqrt{\Delta}) / 2 b$ and therefore $|\tau|^{2}=\left((a-d)^{2}+|\Delta|\right) /(2 b)^{2}$.

As $\tau$ lies in $\mathscr{F}$, we have $|\operatorname{Re}(\tau)| \leq 1 / 2$. This inequality implies $|a-d| \leq|b|$ and hence $|\tau|^{2} \leq\left(b^{2}+|\Delta|\right) /(2 b)^{2}$. By Proposition 1.6.6 of [Bombieri and Gubler 2006] the value $2 h(\tau)$ is at most the logarithmic Mahler measure of $b T^{2}+(a-d) T-c$. So $2 h(\tau) \leq \log \left(|b||\tau|^{2}\right) \leq \log (|b| / 4+|\Delta| /(4|b|))$. The imaginary part of $\tau$ is at least $\sqrt{3} / 2$ and so $|b| \leq \sqrt{|\Delta| / 3}$. As $x \mapsto x+|\Delta| / x$ is decreasing on $[1, \sqrt{|\Delta|}]$, we conclude $2 h(\tau) \leq \log ((1+|\Delta|) / 4) \leq \log |\Delta|$.

Now we use Liouville's inequality to bound the conjugates of $J$ away from zero.

Lemma 6. We have $\log |\sigma(J)| \geq-c_{8} \log |\Delta|$ for any $\sigma: \mathbb{Q}(J) \rightarrow \mathbb{C}$.

Proof. We retain the notation of the proof of Lemma 4 and assume $\left|\tau_{\sigma}-\zeta\right| \leq$ $\left|\tau_{\sigma}-\zeta^{2}\right|$; the reverse case is similar. According to (4), we have

$$
|\sigma(J)|=\left|j\left(\tau_{\sigma}\right)\right| \geq c_{7}\left|\tau_{\sigma}-\zeta\right|^{3} .
$$

We also remark that $\tau_{\sigma} \neq \zeta$ since $\sigma(J) \neq 0=j(\zeta)$. Liouville's inequality, see [Bombieri and Gubler 2006, Theorem 1.5.21], tells us

$$
-\log \left|\tau_{\sigma}-\zeta\right| \leq\left[\mathbb{Q}\left(\tau_{\sigma}, \zeta\right): \mathbb{Q}\right]\left(h\left(\tau_{\sigma}\right)+h(\zeta)+\log 2\right) .
$$

But $\tau_{\sigma}$ and $\zeta$ are imaginary quadratic, so $\left[\mathbb{Q}\left(\tau_{\sigma}, \zeta\right): \mathbb{Q}\right] \leq 4$. Moreover, $h(\zeta)=0$ as $\zeta$ is a root of unity. The bound for $h\left(\tau_{\sigma}\right)$ from Lemma 5 yields

$$
-\log \left|\tau_{\sigma}-\zeta\right| \leq 4 \log (2 \sqrt{|\Delta|}) .
$$

The lemma now follows from $|\Delta| \geq 3$ and (7).

Proof of Theorem 1. We will see soon how to fix $\epsilon$ in terms of the $c_{i}$. By a classical result of Hecke and Heilbronn [Davenport 1980, Chapter 21], there are only finitely many singular moduli whose degree over $\mathbb{Q}$ are bounded by a prescribed constant. So there is no loss of generality if we assume that $D$ is large enough as in Lemma 4 .

We use the previous lemma to bound the first term in (5) from above. Thus

$$
h(J) \leq c_{6} c_{8} \epsilon^{2 / 3} \log |\Delta|+|\log \epsilon| .
$$

We fix $\epsilon$ to satisfy $c_{6} c_{8} \epsilon^{2 / 3}<c_{2} / 2$, where $c_{2}$ comes from the height lower bound in Lemma 3. With this choice we conclude that $|\Delta|$ is bounded from above by an absolute constant. By Lemma 5 and Northcott's theorem [Bombieri and Gubler 2006, Theorem 1.6.8] there are only finitely many possible $\tau_{\sigma}$ and thus only finitely many possible $J$. 


\section{Proof of Theorem 2}

We begin by stating a special case of David and Hirata-Kohno's deep lower bound for linear forms in $n$ elliptic logarithms if $n=2$ and when the elliptic logarithms are periods. Let $E$ be an elliptic curve defined over a number field in $\mathbb{C}$. We fix a Weierstrass equation for $E$ with coefficients in the said number field and a Weierstrass- $\wp$ function that induces a uniformization $\mathbb{C} \rightarrow E(\mathbb{C})$. This is a group homomorphism whose kernel $\omega_{1} \mathbb{Z}+\omega_{2} \mathbb{Z}$ is a discrete subgroup of $\mathbb{C}$. We start numbering constants anew.

Lemma 7. Let $d \geq 1$. There exists a constant $c_{1}>0$ depending on $E, d$, the choice of Weierstrass equation, and the choice $\omega_{1,2}$ with the following property. Suppose $\alpha, \beta \in \mathbb{C}$ are algebraic over $\mathbb{Q}$ of degree at most $d$ and $\max \{1, h(\alpha), h(\beta)\} \leq \log B$ for some real number $B>0$. If $\alpha \omega_{1}+\beta \omega_{2} \neq 0$, then

$$
\log \left|\alpha \omega_{1}+\beta \omega_{2}\right| \geq-c_{1} \log B .
$$

Proof. This follows from [David and Hirata-Kohno 2009, Theorem 1.6].

In our application, the $\log B$ from (8) will be approximately $\log |\Delta|$ and will compete directly with the logarithmic lower bound of Lemma 3. It is thus essential that David and Hirata-Kohno's inequality is logarithmic in $B$. A worse dependency, such as $-c_{1}(\log B)(\log \log B)$, would not suffice.

We further distill this result into a formulation adapted to our application.

Lemma 8. Let $\eta \in \mathbb{H}$ be such that $j(\eta)$ is an algebraic number. There exists a constant $c_{2}>0$ which may depend on $\eta$ with the following property. If $\tau \neq \eta \in \mathbb{U}$ is imaginary quadratic with $\max \{1, h(\tau)\} \leq \log B$ for some real number $B>0$, then

$$
\log |\tau-\eta| \geq-c_{2} \log B \text {. }
$$

Proof. The algebraic number $j(\eta)$ is the $j$-invariant on an elliptic curve as introduced before Lemma 7. We may assume that the periods $\omega_{1,2}$ satisfy $\eta=\omega_{2} / \omega_{1}$. As $\tau \neq \eta$, Lemma 7 with $\alpha=\tau$ and $\beta=-1$ implies $\log \left|\tau \omega_{1}-\omega_{2}\right| \geq-c_{1} \log B$. We subtract $\log \left|\omega_{1}\right|$ and obtain $\log |\tau-\eta| \geq-c_{1} \log B-\log \left|\omega_{1}\right|$. This lemma follows with an appropriate $c_{2}$ as $\log B \geq 1$.

Let us suppose that $\Gamma, F$, and $C$ are as in Theorem 2. We recall that $\phi$ is the natural morphism $Y_{\Gamma} \rightarrow Y(1)$ and we may regard it as an element in the function field of $X$. We abbreviate $X=X_{\Gamma}$. In the following, we enlarge $F$ to a number field for which $X(F)$ contains $C$ and all poles of $\phi$.

By hypothesis there is a $P_{0} \in C$ that is not a cusp of $Y_{\Gamma}$. We write $J_{0} \in F$ for the value of $\phi$ at $P_{0}$. The Riemann-Roch theorem provides a nonconstant, rational function $\psi \in F\left[X \backslash\left\{P_{0}\right\}\right]$ that vanishes at the poles of $\phi$. As $\psi$ is regular outside of $P_{0}$, it must have a pole at $P_{0}$. 
The functions $\phi$ and $\psi^{-1}$ are algebraically dependent, i.e., there is an irreducible polynomial $R \in F[U, V]$ with $R\left(\phi, \psi^{-1}\right)=0$. We observe $\operatorname{deg}_{U} R>0$.

Lemma 9. There exists a constant $c_{5} \in(0,1]$ which depends only on $R$ with the following property. Let $K \supset F$ be a number field and $|\cdot|$ an absolute value on $K$ that extends the Archimedean absolute value on $\mathbb{Q}$. If $u \in K$ and $v \in K \backslash\{0\}$ with $R(u, v)=0,|v|<c_{5}$, and $u \neq J_{0}$, then $\log \left|u-J_{0}\right|<(\log |v|) /\left(2 \operatorname{deg}_{U} R\right)$.

Proof. In this proof, $c_{3,4}>0$ depend only on $R$. We put $e=\operatorname{deg}_{U} R$ and write $R=r_{0}+\left(U-J_{0}\right) r_{1}+\cdots+\left(U-J_{0}\right)^{e} r_{e}$, with $r_{i} \in F[V]$ and $r_{e} \neq 0$.

By construction, the poles of $\phi$ are among the poles of $\psi^{-1}$. So $\phi$, and thus $\phi-J_{0}$, are integral over the ring $F\left[\psi^{-1}\right]$, see for example [Matsumura 1989, Theorem 10.4]. Hence, $r_{e}$ is constant and without loss of generality we may assume $r_{e}=1$. Next we claim $r_{i}(0)=0$ if $0 \leq i \leq e-1$. If this were not the case, we could find $J_{0}^{\prime} \neq J_{0}$ with $R\left(J_{0}^{\prime}, 0\right)=0$. This is impossible by our choice of $\psi$. Therefore,

$$
R=V Q+\left(U-J_{0}\right)^{e}
$$

for some $Q \in F[U, V]$ with $\operatorname{deg}_{U} Q \leq e-1$.

Now let $u$ and $v$ be as in the hypothesis; we will see how to fix $c_{5} \in(0,1]$ below. We have $\left|u-J_{0}\right|^{e}=|v Q(u, v)|$ and $|v Q(u, v)| \leq c_{3} \max \{1,|u|\}^{e-1}$ as $|v| \leq 1$. If $|u| \geq \max \left\{1,2\left|J_{0}\right|\right\}$, then $\left|u-J_{0}\right| \geq|u|-\left|J_{0}\right| \geq|u| / 2$ and so $|u|^{e} \leq 2^{e} c_{3}|u|^{e-1}$. We find $|u| \leq 2^{e} c_{3}$. In this case, $\left|u-J_{0}\right|^{e}=|v Q(u, v)| \leq c_{4}|v|$ for some $c_{4} \geq 1$. After adjusting, $c_{4}$ the same bound holds if $|u|<\max \left\{1,2\left|J_{0}\right|\right\}$. We set $c_{5}=c_{4}^{-2}$ and observe $c_{4}|v|<|v|^{1 / 2}$ if $|v|<c_{5}$. Thus $\left|u-J_{0}\right|^{e} \leq|v|^{1 / 2}<1$ and the lemma follows on taking the logarithm.

Let us now prove Theorem 2. For this we must verify that a set $M \subset X(\bar{F})$ of special points that is quasi-algebraic-integral with respect to $C$ is finite. By definition, $M$ cannot contain the pole of $\psi$ and without loss of generality we may assume that $M$ does not contain its zeros either. Finally, we may assume that $J_{0} \notin \phi(M)$. Let $\lambda \in F \backslash\{0\}$ satisfy $\lambda \psi(M) \subset \mathcal{O}_{\bar{F}}$. To simplify notation we replace $\lambda \psi$ by $\psi$ and adapt $R$ accordingly.

We will use $c_{6}, c_{7}, \ldots$ to denote positive constants that may depend on $\Gamma, F, C$, and $M$. Suppose $P \in M$ and let $K \subset \bar{F}$ be a number field containing $F$ and the values $\psi(P), \phi(P)$. Then, as usual,

$$
\begin{aligned}
h(\psi(P)) & =\frac{1}{[K: \mathbb{Q}]} \sum_{|\sigma(\psi(P))|>1} \log |\sigma(\psi(P))| \\
& =\frac{1}{[K: \mathbb{Q}]}\left(\sum_{1<|\sigma(\psi(P))| \leq c_{5}^{-1}} \log |\sigma(\psi(P))|+\sum_{|\sigma(\psi(P))|>c_{5}^{-1}} \log |\sigma(\psi(P))|\right) \\
& \leq-\log c_{5}+\frac{1}{[K: \mathbb{Q}]} \sum_{|\sigma(\psi(P))|>c_{5}^{-1}} \log |\sigma(\psi(P))|,
\end{aligned}
$$


where the sums run over field embeddings $\sigma: K \rightarrow \mathbb{C}$. Say $J=\phi(P) \in K$, then $R\left(J, \psi(P)^{-1}\right)=0$. We apply Lemma 9 to $u=J$ and $v=\psi(P)$ to obtain

$$
h(\psi(P)) \leq c_{6}\left(1+\frac{1}{[K: \mathbb{Q}]} \sum_{\left|\sigma\left(J-J_{0}\right)\right|<1}-\log \left|\sigma\left(J-J_{0}\right)\right|\right) .
$$

We have already seen that $R$ is not divisible by a linear polynomial in the variable $V$. So, [Bilu and Masser 2006, Proposition 5] and $R\left(J, \psi(P)^{-1}\right)=0$ allow us to bound $h(J)$ from above linearly in terms of $h\left(\psi(P)^{-1}\right)=h(\psi(P))$. Together with (9) we get

$$
\begin{aligned}
h(J) & \leq c_{7}\left(1+\frac{1}{[K: \mathbb{Q}]} \sum_{\left|\sigma\left(J-J_{0}\right)\right|<1}-\log \left|\sigma\left(J-J_{0}\right)\right|\right) \\
& \leq c_{7}\left(|\log \epsilon|+\frac{1}{[K: \mathbb{Q}]} \sum_{\left|\sigma\left(J-J_{0}\right)\right|<\epsilon}-\log \left|\sigma\left(J-J_{0}\right)\right|\right)
\end{aligned}
$$

for any $\epsilon \in(0,1 / 2]$.

The points in $M$ are special, so $J$ is a singular modulus. An elliptic curve attached to $J$ has complex multiplication by an order with discriminant $\Delta<0$. As in the previous section, we will find an upper bound for $|\Delta|$.

For any embedding $\sigma: K \rightarrow \mathbb{C}$ we fix $\tau_{\sigma} \in \mathscr{F}$ with $j\left(\tau_{\sigma}\right)=\sigma(J)$. We now proceed as near (4) and apply [Lang 1987, Chapter 3, Theorem 2]. If $\epsilon$ is sufficiently small and if $\left|\sigma\left(J-J_{0}\right)\right|<\epsilon$, then

$$
\left|\sigma\left(J-J_{0}\right)\right| \geq \begin{cases}c_{8}\left|\tau_{\sigma}-\eta_{\sigma}\right|^{3} & \text { if } J_{0}=0, \\ c_{8}\left|\tau_{\sigma}-\eta_{\sigma}\right|^{2} & \text { if } J_{0}=1728, \\ c_{8}\left|\tau_{\sigma}-\eta_{\sigma}\right| & \text { else. }\end{cases}
$$

for some $\eta_{\sigma} \in \overline{\mathscr{F}}$ with $j\left(\eta_{\sigma}\right)=\sigma\left(J_{0}\right)$. It is harmless that there are 2 choices for $\eta_{\sigma}$ on the boundary of $\overline{\mathscr{F}}$. We note that $\eta_{\sigma}$ depends only on the base point $J_{0}$ and that $\tau_{\sigma}$ is imaginary quadratic. Thus Lemma 8 and the height bound for $\tau_{\sigma}$ in Lemma 5 yield $\log \left|\sigma\left(J-J_{0}\right)\right| \geq-c_{9} \log |\Delta|$. We use this inequality and (10) to bound

$$
h(J) \leq c_{10}\left(\log |\epsilon|+\log |\Delta| \frac{\#\left\{\sigma: K \rightarrow \mathbb{C} ;\left|\sigma\left(J-J_{0}\right)\right|<\epsilon\right\}}{[K: \mathbb{Q}]}\right)
$$

for all $\epsilon \in(0,1 / 2]$.

The rest of the proof resembles the proof of Theorem 1. Indeed, we may assume that $[\mathbb{Q}(J): \mathbb{Q}]$ is sufficiently large and as in Lemma 4 we use equidistribution to prove that $[K: \mathbb{Q}]^{-1} \#\left\{\sigma: K \rightarrow \mathbb{C} ;\left|\sigma\left(J-J_{0}\right)\right|<\epsilon\right\}$ is bounded from above linearly by a fixed power, derived from (11), of $\epsilon$. Finally, we again use the height lower bound of Lemma 3 to fix an appropriate $\epsilon$ which leads to a bound on $|\Delta|$. As before, this leaves us with only finitely many possibilities for $J=\phi(P)$. 


\section{References}

[Baker et al. 2008] M. Baker, S.-i. Ih, and R. Rumely, "A finiteness property of torsion points", Algebra Number Theory 2:2 (2008), 217-248. MR 2009g:11078 Zbl 1182.11030

[Bilu and Masser 2006] Y. F. Bilu and D. Masser, “A quick proof of Sprindzhuk's decomposition theorem", pp. 25-32 in More sets, graphs and numbers, edited by E. Györi et al., Bolyai Soc. Math. Stud. 15, Springer, Berlin, 2006. MR 2007b:11031 Zbl 1147.11018

[Bilu et al. 2013] Y. Bilu, D. Masser, and U. Zannier, "An effective "theorem of André" for $C M$-points on a plane curve", Math. Proc. Cambridge Philos. Soc. 154:1 (2013), 145-152. MR 3002589

[Bombieri and Gubler 2006] E. Bombieri and W. Gubler, Heights in Diophantine geometry, New Mathematical Monographs 4, Cambridge Univ. Press, 2006. MR 2007a:11092 Zbl 1115.11034

[Clozel and Ullmo 2004] L. Clozel and E. Ullmo, "Équidistribution des points de Hecke", pp. 193254 in Contributions to automorphic forms, geometry, and number theory, edited by H. Hida et al., Johns Hopkins Univ. Press, Baltimore, MD, 2004. MR 2005f:11090 Zbl 1068.11042

[Colmez 1998] P. Colmez, "Sur la hauteur de Faltings des variétés abéliennes à multiplication complexe”, Compositio Math. 111:3 (1998), 359-368. MR 99e:11074 Zbl 0918.11025

[Davenport 1980] H. Davenport, Multiplicative number theory, 2nd ed., Graduate Texts in Mathematics 74, Springer-Verlag, New York, 1980. MR 82m:10001 Zbl 0453.10002

[David and Hirata-Kohno 2009] S. David and N. Hirata-Kohno, "Linear forms in elliptic logarithms", J. Reine Angew. Math. 628 (2009), 37-89. MR 2010c:11084 Zbl 1169.11034

[Dorman 1988] D. R. Dorman, "Special values of the elliptic modular function and factorization formulae”, J. Reine Angew. Math. 383 (1988), 207-220. MR 89k:11026 Zbl 0626.10022

[Duke 1988] W. Duke, "Hyperbolic distribution problems and half-integral weight Maass forms", Invent. Math. 92:1 (1988), 73-90. MR 89d:11033 Zbl 0628.10029

[Gross and Zagier 1985] B. H. Gross and D. B. Zagier, "On singular moduli”, J. Reine Angew. Math. 355 (1985), 191-220. MR 86j:11041 Zbl 0545.10015

[Habegger 2010] P. Habegger, "Weakly bounded height on modular curves", Acta Math. Vietnam. 35:1 (2010), 43-69. MR 2011g:11124 Zbl 1253.11070

[Lang 1987] S. Lang, Elliptic functions, 2nd ed., Graduate Texts in Mathematics 112, Springer, New York, 1987. MR 88c:11028 Zbl 0615.14018

[Matsumura 1989] H. Matsumura, Commutative ring theory, 2nd ed., Cambridge Studies in Advanced Mathematics 8, Cambridge Univ. Press, 1989. MR 90i:13001 Zbl 0666.13002

[Nakkajima and Taguchi 1991] Y. Nakkajima and Y. Taguchi, "A generalization of the ChowlaSelberg formula”, J. Reine Angew. Math. 419 (1991), 119-124. MR 92g:11113 Zbl 0721.11045

[Poonen 2001] B. Poonen, "Spans of Hecke points on modular curves", Math. Res. Lett. 8:5-6 (2001), 767-770. MR 2002k:11092 Zbl 1081.11043

[Serre 1997] J.-P. Serre, Lectures on the Mordell-Weil theorem, 3rd ed., Aspects of Mathematics 15, Friedr. Vieweg \& Sohn, Braunschweig, 1997. MR 2000m:11049 Zbl 0863.14013

[Silverman 1986] J. H. Silverman, "Heights and elliptic curves", pp. 253-265 in Arithmetic geometry (Storrs, Conn., 1984), edited by G. Cornell and J. H. Silverman, Springer, New York, 1986. MR 861979 Zbl 0603.14020

Communicated by Joseph Silverman

Received 2014-03-19 Revised 2015-05-30 Accepted 2015-07-15

philipp.habegger@unibas.ch Departement Mathematik und Informatik, Universität Basel, Spiegelgasse 1, CH-4051 Basel, Switzerland 


\section{Algebra \& Number Theory}

msp.org/ant

\section{EDITORS}

MANAGING EDITOR

Bjorn Poonen

Massachusetts Institute of Technology

Cambridge, USA

\author{
EDITORIAL BOARD CHAIR \\ David Eisenbud \\ University of California \\ Berkeley, USA
}

BOARD OF EDITORS

Georgia Benkart

Dave Benson

Richard E. Borcherds

John H. Coates

J-L. Colliot-Thélène

Brian D. Conrad

Hélène Esnault

Hubert Flenner

Sergey Fomin

Edward Frenkel

Andrew Granville

Joseph Gubeladze

Roger Heath-Brown

Craig Huneke

Kiran S. Kedlaya

János Kollár

Yuri Manin

Philippe Michel
University of Wisconsin, Madison, USA

University of Aberdeen, Scotland

University of California, Berkeley, USA

University of Cambridge, UK

CNRS, Université Paris-Sud, France

Stanford University, USA

Freie Universität Berlin, Germany

Ruhr-Universität, Germany

University of Michigan, USA

University of California, Berkeley, USA

Université de Montréal, Canada

San Francisco State University, USA

Oxford University, UK

University of Virginia, USA

Univ. of California, San Diego, USA

Princeton University, USA

Northwestern University, USA

École Polytechnique Fédérale de Lausanne
Susan Montgomery

Shigefumi Mori

Raman Parimala

Jonathan Pila

Anand Pillay

Victor Reiner

Peter Sarnak

Joseph H. Silverman

Michael Singer

Vasudevan Srinivas

J. Toby Stafford

Ravi Vakil

Michel van den Bergh

Marie-France Vignéras

Kei-Ichi Watanabe

Efim Zelmanov

Shou-Wu Zhang
University of Southern California, USA

RIMS, Kyoto University, Japan

Emory University, USA

University of Oxford, UK

University of Notre Dame, USA

University of Minnesota, USA

Princeton University, USA

Brown University, USA

North Carolina State University, USA

Tata Inst. of Fund. Research, India

University of Michigan, USA

Stanford University, USA

Hasselt University, Belgium

Université Paris VII, France

Nihon University, Japan

University of California, San Diego, USA

Princeton University, USA

\section{PRODUCTION}

production@msp.org

Silvio Levy, Scientific Editor

See inside back cover or msp.org/ant for submission instructions.

The subscription price for 2015 is US $\$ 255 /$ year for the electronic version, and $\$ 440 /$ year ( $+\$ 55$, if shipping outside the US) for print and electronic. Subscriptions, requests for back issues and changes of subscribers address should be sent to MSP.

Algebra \& Number Theory (ISSN 1944-7833 electronic, 1937-0652 printed) at Mathematical Sciences Publishers, 798 Evans Hall \#3840, c/o University of California, Berkeley, CA 94720-3840 is published continuously online. Periodical rate postage paid at Berkeley, CA 94704, and additional mailing offices.

ANT peer review and production are managed by EditFLOW ${ }^{\circledR}$ from MSP.

\section{PUBLISHED BY}

- mathematical sciences publishers

nonprofit scientific publishing

http://msp.org/

(C) 2015 Mathematical Sciences Publishers 


\section{Algebra \& Number Theory}

Volume $9 \quad$ No. $7 \quad 2015$

Singular moduli that are algebraic units

PHILIPP HABEGGER

Irreducibility of the Gorenstein loci of Hilbert schemes via ray families

GIANFRANCO CASNATI, JOACHIM JELISIEJEW and ROBERTO NOTARI

$p$-adic heights of Heegner points on Shimura curves

DANIEL DISEGNI

Calculabilité de la cohomologie étale modulo $\ell$

David A. Madore and FABrice ORgogozo 